\title{
Facharztprüfung - was gilt?
}

Ch. Hänggeli, Geschäftsleiter Sekretariat Aus-, Weiter- und Fortbildung (AWF)

Der Zentralvorstand setzt per 1. Januar 2003 auch die Facharztprüfungen in Allergologie und klinischer Immunologie, Dermatologie und Venerologie, Infektiologie, Neurologie, Orthopädischer Chirurgie, Pharmazeutischer Medizin, Physikalischer Medizin und Rehabilitation, Pneumologie, Neonatologie sowie Neuropädiatrie mit eliminatorischer Wirkung in Kraft.

Per 1. Januar 2003 hat der Zentralvorstand in zehn weiteren Fachgebieten die eliminatorische Wirkung der Facharztprüfung in Kraft gesetzt. Damit wird jetzt bereits für mehr als die Hälfte aller Facharzttitel das Bestehen der Facharztprüfung gefordert, selbstverständlich unter Vorbehalt der anwendbaren Übergangsbestimmungen.

Nachfolgend publizieren wir wiederum den aktuellen Stand, in welchen Fachgebieten für die Erlangung des Facharzttitels das Bestehen und in welchen lediglich die Teilnahme an einer Facharztprüfung gefordert ist.

\section{Die bestandene Facharztprüfung ist Voraussetzung für die Erteilung des Facharzttitels}

In folgenden Fachgebieten werden Facharzttitel und Schwerpunkte - unter Vorbehalt der nachstehenden Ausnahmen - nur noch nach bestandener Facharztprüfung erteilt:

\begin{tabular}{lr} 
Facharzttitel & Inkraftsetzungstermin \\
\hline Allergologie und klinische Immunologie & 1.1 .2003 \\
\hline Allgemeinmedizin & 1.1 .2000 \\
\hline Anästhesiologie & 2.4 .1986 \\
\hline Arbeitsmedizin & 1.1 .2001 \\
\hline Chirurgie (inkl. Basisexamen) & 1.1 .1999 \\
\hline Dermatologie und Venerologie & 1.1 .2003 \\
\hline Endokrinologie / Diabetologie & 1.1 .2001 \\
\hline Gastroenterologie & 1.1 .2000
\end{tabular}

Gynäkologie und Geburtshilfe

1.1 .1999

\begin{tabular}{lc}
\hline Hämatologie & 1.1 .2001 \\
\hline Infektiologie & 1.1 .2003 \\
\hline Innere Medizin & 1.1 .1999 \\
\hline Intensivmedizin & 1.1 .2001 \\
\hline Kardiologie & 1.1 .1999 \\
\hline Kinderchirurgie & 2.4 .1986 \\
\hline Kinder- und Jugendpsychiatrie und & 1.1 .2002 \\
\hline -psychotherapie & \\
\hline
\end{tabular}

Medizinische Onkologie 1.1 .2001

Medizinische Onkologie 2.4 .1986

Neurochirurgie

1.1 .2003

Neurologie 2.4 .1986

Nuklearmedizin

1.1 .2002

Ophthalmologie

1.1 .2001

\begin{tabular}{ll}
\hline ORL (ohne Hals- und Gesichtschirurgie) & 1.1 .2001 \\
\hline Orthopädische Chirurgie & 1.1 .2003
\end{tabular}

\begin{tabular}{ll} 
Orthopädische Chirurgie & 1.1 .2003 \\
\hline Pathologie & 1.1 .1999
\end{tabular}

Pharmazeutische Medizin $\quad 1.1 .2003$

Physikalische Medizin und Rehabilitation $\quad 1.1 .2003$

Pneumologie $\quad 1.1 .2003$

Psychiatrie und Psychotherapie $\quad 1.1 .2001$

Radiologie 2.4 .1986

Radio-Onkologie / Strahlentherapie $\quad 2.4 .1986$

Rechtsmedizin $\quad 1.1 .2001$

Rheumatologie $\quad 1.1 .2001$

Tropen- und Reisemedizin $\quad 1.1 .2001$

Urologie

1.1 .2002

Schwerpunkte Inkraftsetzungstermin

\begin{tabular}{ll}
\hline Neonatologie & 1.1 .2003 \\
\hline Neuropädiatrie & 1.1 .2003 \\
\hline Pädiatrische Kardiologie & 1.1 .2001 \\
\hline
\end{tabular}


Tabelle 1

Muss ich die Facharztprüfung bestehen?

Inkraftsetzungstermin

Allergologie und klinische Immunologie

Allgemeinmedizin

Anästhesiologie

Arbeitsmedizin

Chirurgie (inkl. Basisexamen)

Dermatologie und Venerologie

Endokrinologie / Diabetologie

Gastroenterologie

Gynäkologie und Geburtshilfe

Hämatologie

Infektiologie

Innere Medizin

Intensivmedizin

Kardiologie

Kinderchirurgie

Kinder- und Jugendpsychiatrie und -psychotherapie

Medizinische Onkologie

Neonatologie

Neurochirurgie

Neurologie

Neuropädiatrie

Nuklearmedizin

Ophthalmologie

ORL (ohne Hals- und Gesichtschirurgie)

Orthopädische Chirurgie

Pathologie

Pädiatrische Kardiologie

Pharmazeutische Medizin

Physikalische Medizin und Rehabilitation

Pneumologie

Psychiatrie und Psychotherapie

Radiologie

Radio-Onkologie / Strahlentherapie

Rechtsmedizin

Rheumatologie

Tropen- und Reisemedizin

\section{Urologie}

alle anderen Fachgebiete

$\mathrm{B}=$ Bestehen; $\mathrm{T}$ = Teilnehmen

Die gesamte Weiterbildungsdokumentation (mit den aktuellen Prüfungsterminen) ist auf dem Internet unter www.fmh.ch/awf abrufbar. Die Prüfungstermine werden auch in der Schweizerischen Ärztezeitung publiziert. Bei allfälligen Rückfragen steht Ihnen das Sekretariat Aus-, Weiter- und Fortbildung (AWF) der FMH gerne zur Verfügung. Adresse: Postfach 293, Elfenstrasse 18, 3000 Bern 16, Tel. 03135911 11, Fax 03135911 12, E-Mail: diplome@hin.ch.

\section{Übergangsbestimmungen}

Ausnahmen gelten für Kandidatinnen und Kandidaten, welche eine der beiden folgenden Bedingungen erfüllen:

- Wer vor dem Inkraftsetzungstermin bereits an einer Facharztprüfung (ganz oder teilweise) teilgenommen hat, muss keine weitere Prüfung absolvieren.

- Wer die Weiterbildung innert zweier Jahre nach dem Inkraftsetzungstermin abgeschlossen hat, benötigt für die Titelerteilung lediglich eine Teilnahmebestätigung über die absolvierte Facharztprüfung. Achtung: Sämtliche Voraussetzungen müssen innert zweier Jahre nach dem Inkraftsetzungstermin erfüllt sein (inkl. komplette Facharztprüfung, ausser wenn die Fachgesellschaft die Zulassung zum 2. Teil wegen ungenügendem 1 . Teil verweigert)!

\section{Nur die Teilnahme an der Facharzt- prüfung ist Voraussetzung für die Erteilung des Facharzttitels}

In allen übrigen Fachgebieten ist für die Erteilung des Facharzttitels bzw. des Schwerpunktes lediglich eine Teilnahmebestätigung beizubringen. Das Bestehen dieser Prüfungen wird frühestens ab 1. Januar 2004 gefordert, analog zu den oben beschriebenen Fachgebieten mit einer 2jährigen Übergangsfrist. 